\title{
II. Ueber die neueren Fortschritte der chirurgischen Technik.
}

Von Oberstabsarzt Prof. Dr. A. Köhler in Berlin.

\section{Specieller Theil.2)}

Die durch Vervollkommnung der Untersuchungsmethoden möglich gewordenen Fortschritte liessen sich, wie wir gesehen liaben, am besten an der Hirnchirurgie demonstriren; dem entspricht es nur, wenn wir auch hinsichtlich der chirurgischen Technik auf diesem Gebiete die zalllreichsten neuen Vorschläge antreffen. Mag die Hirnchirurgie von einigen und an einzelnen Stellen schon zu schnell vorwärts getrieben sein, mag deshalb hier und da (siehe Krönlein, Correspondenzblatt 1890, No. 1 und 2) eine Warnung, ein "kalter Wasserstrahl“ nöthig und nützlich sein - im allgemeinen ist dieser Zweig der Chirurgie in stetem Wachsen begriffen; jeder Tag bringt neue Vorschläge, und durch die gemeinsame Arbeit der verschiedenen medicinischen Disciplinen, durch die Leichtigkeit, mit welcher heute die Erfahrungen der ganzen wissenschaftlichen Welt gesammelt und kritisch verwerthet werden können, ist die Prüfung dieser zahlreichen neuen Vorschläge und die Entscheidung darüber, ob sie einen wirklichen und bleibenden Vortheil bedeuten, immer leichter geworden. Man sollte meinen, dass damit auch eine gewisse Stetigkeit des Fortschrittes gewährleistet sei und dass jene grossen Wechsel in der Werthschätzung bestimmter Eingriffe, wie z. B. der Trepanation, welche bald in den Himmel erhoben, bald als Verbrechen bezeichnet wurde, hente ausgeschlossen seien. Wir wollen uns dieser Meinung mit aller Vorsicht anschliessen, wenn wir auch die Möglichkeit eines Wechsels im einzelnen zugeben mïssen. Wie das Schicksal der Blutentziehungen der Trepanation ${ }^{3}$ ), der Transfusion ${ }^{4}$, verschiedener Anästhetica und vieler moderner therapeutischer Vorschläge lehrt, können wir manches Neue und begeistert Empfohlene nicht sofort als fest gesicherten Besitz betrachten.

Die neueren Fortschritte der chirurgischen Technik bei der Behandlung der Verletzungen, Entzündungen, Neu- und Missbildungen am Kopfe bestehen hauptsächlich in der Art der Eingriffe, der Trepanation; der Methoden, der Instrumente, der Vermeidung von Schädeldefecten, der Deckung dieser Defocte (Cranioplastik) und denjenigen Methoden, welche bei der Behandlung der Mikrocephalie, des Hydrocephalus und in einzelnen Fällen bei Geisteskrankheiten empfohlen sind. - Die Behandlung der Kopfverletzungen erfordert keine besondere

l) Im Harn konnte ich das Phenol nach der Injection prompt nach= weisen (sowohl mit Bromwasser als mit Milton, Plugge u, s, w.), selbstverständlich nach vorheriger Destillation des Harnes.

2) Siehe diese Wochenschrift No. 1, S. 2.

3) Vergl. u. a. Fronmueller, Ueber die Wandlungen der 'Trepanation. Erlangen 1892.

4) Siehe den Abschnitt uber Transfusion im Kriegs-Sanitätsbericht 1890 , III A, 342 und 171 . 
Technik; sie erfolgt nach den Grundsätzen der allgemeinen Wundbehandlung, solange die Schädelkapsel unversehrt geblieben ist. Man wird, eventuell nach Abtragen gequetschter Ränder, nähell, wo überhaupt die Möglichkeit antiseptischer Säuberung vorliegt. Wie segensreich auf diesem Gebiete Anti- und Aseptik gewirkt haben, das wird durch die von allen Klinikern beobachtete auffallende Abnahme in der Häufigkeit von Hirnabscessen nach Kopfverletzungen bewiesen. - Wie man verfahren soll, wenn die Schädelkapsel verletzt ist, darüber sind die Meinungen noch getheilt. Handelt es sich um einen offenen Bruch mit Depression, dann wird liemand säumen, diese zu beseitigen, Splitter zu entfernen u.s.w. (s. u. a. Marchand, Soc. de chir. Bd. XVIII). Freilich schützt auch dieses primäre Débridement nicht imnier vor den Exostosell, den Folgen unregelmässiger Callusbildung im Verlaufe der Heilung der Schädelbrüche, wie z. B. der von Berger beobachtete Fall (Sem. méd. 1892, S. 415) beweist; doch sind das immerhin Ausnahmen, und wir haben kein besseres Mittel, wenigstens in der Regel die Bildung schlechter Knochennarben und der darauf folgenden Reizungs- und Lähmungszustände, am häufigsten also die traumatische Rindenepilepsie zu vermeiden. Zweifelhafter ist der Eingriff bei einer Depression ohne Wunde; hier kann auch einmal die Vitrea stark gesplittert, die Meningea media verletzt sein u. s. w. Dann werden aber deutliche Symptome dieser Complicationen bestimmte energische Eingriffe erfordern. Bei allen Depressionen, auch bei denjenigen, welche keine allgemeinen oder Herderscheinungen von Seiten des Gehirns verursachen, ausnahmslos trepaniren zu wollen, ist wohl trotz des "Schutzes der Antiseptik" niclit zu rechtfertigen. Man weiss ausserdem, dass das Gehirll sich an mässigen Druck gewöhnt.

Die grossartigen Erfolge, welche auf dem Gebiete der lach Jackson benannten Rindenepilepsie in den letzten Jahrzehntell erzielt sind, können wir liier nur kurz erwähnen. Diejenigen Fälle, in denen dabei vollständige Heilung eintrat, sind ja selten; aber imposant ist die Zahl derer, welche bedeutend gebessert wurden. Sie waren fast alle vor der Operation lıïlflos, zı keinem Erwerbe fähig, in den freien Intervallen in ewiger Angst vor dem unheimlichell Anfall, bei welchen sie mit klarem Bewusstsein fühlten und sahen, dass ein Theil ihres Körpers nach dem anderen ihrem Willen nicht mehr gehorchte und in schmerzhafte Zuckungen verfiel, bis mit der Ausbreitung des Anfalles auch das Bewusstsein erloscll. Hier ist Grossartiges geleistet, und die Belıandlung der traunıatischen Rindenepilepsie vorläufig nur dieser Form - kann als gesichertes chirurgisehes Eigenthun eingetragen werden. Ist die Krankheit Folge einer Verletzung, dann liegt es nahe, die Narbe aufzusuchen und bei der Trepanation von ihr auszugehell; das mag in vielen Fällen genügen, aber nicht immer. Oft genug entspriclit die äussere Narbe denl ilnneren Schädlichkeiten nicht; es ist deshalb sicherer, sich all die Symptome zu halten und auf Grund der'selben nach den oben allgegebenell Methoden die Stelle zu bestimmen, an welcher der Schädel geöffnet werden soll. In dem bekannten Falle voul Bennet und Godlee, sowie in mehreren anderen, z. B. dein Falle von Exostosen nach Säbelhieb auf der v. Bardeleben'schen Klinilk, bei welchem der Hieb schräg von vorn llach hillten durch Weichtheile und Knochen gedrungen war, wurde mit Vortheil dieser. Weg eingeschlagen. Wenn man eine der Methoden der temporären Resection hierbei anwendet, einen grossen Weichtheilknochenlappen bildet, welcher nachher wieder eingelegt wird, dann ist natürlich eine genaue Localisation vol dem Eillgriff nicht nöthig. Ob man nun Exostosell, Verwachsungen, alte Blutgerinnsel, Cystell, Tumoren findet - wenll die typische Rindenepilepsie vorhanden war, muss der krankhafte Zustand in oder dicht unter der Rindensubstanz zu findell seill. Zuweilen fand man nichts und sah doch dic Krämpfe nach dem Eingriffe schwinden (z. B. Fall Benda, XX. Chirurgencongress 1890). Es ist schwer, für diese Fälle eine genügende Erklärung zu finden; am nächsten liegt es noch, einen günstigen Einfluss der Trepanation auf makroskopisch nicht erkennbarc Circulationsverlä̈ltnisse in der Rindensubstanz allzunehmen.

Im allgemeinen sind die Erfolge bei Hirnabscessen und Tumoren nicht so günstig; volle Heilungen sind noch seltener, besonders bei Abscessen, wolche in grösserer 'Tiefe liegen und bei denen mall die Weiterverbreitulg und den schlimmen Einfluss auf die allgemeine Infection weniger in der Hand hat. Abscesse und Tumoren können ausserdem bei der Localisation irreführen; an irgend einer Stelle in der Nähe ihrer Peripherie befinden sich motorische Centren; von diesen gehen die einzigen Heerdsymptome aus, welche die Läsion hervorruft. Wenn man nun hier trepanirt, daun kann es vorkommen, dass die seitlich und in der Tiefe gelegene Ursache der Krankheit nicht gefunden wird. Vorläufig können wir nur die in der Nähe der Rindensubstanz gelegenen Heerde operativ angreifen; wie häufig aber z. B. die tiefer gelegenen oder mehrfachen Tumorel im Gehirn sind, das lehren die
Zusammenstellungen über die bei Sectionen gefundenen Hirntumoren; dass nur ein kleiner Theil von diesen zu operiren gewesen wäre, fand u. a. Good hart (Pathol. Soc. of London, Febr. 1886); nach Seydel (XXI. Chirurgencongress 1892) wären von 500 Hirngeschwülsten, welche bei Sectionen gefunden wurdell, mur 11, also $2 \%$, zu operiren gewesen. - Wie man Hirnabscesse nicht behandeln soll, lehrt ein Fall von Mas (Valencia) (Ref. in der Deutschen Zeitschrift für Chirurgie Bd. 39, S. 419), bei welchem der Abscess entleert, ausgespült und dann Dura und Haut - vernäht wurden. Der Kranke lebte noch ca. acht Tage. - Der neueste, den Hirnabscess und seine Behandlung betreffellde Vol-schlag ist der von M. Schmidt (Langellbeck's Archiv Bd. 45): an del vermutheten Stelle wird der Schädel nur angebohrt und durch diese Oeffnung die diagnostische Gehirnpunction ausgefïhrt. Das Resultat derselben ist für das weitere Vorgehen maassgebend.

In dem Instrumentarium zur Trepanation behaupten sich die alten Hand- und Bogentrepane neben den neu erfundenen und empfohlenen Meisseln, Sägen und complicirteren Apparaten. Im allgemeinen haben Hammer- und Meissel trotz der vielfacl geäusserten Besorgniss, durch "Verhämmern" des Gehirns Schadell zu stiften, eine immer weitere Verwendung g'efunden; diejenigen aber, welche infolge dessen die Trepankrone aus den chirurgischell Bestecks ganz verbannen wollen, haben doch noch zahlreiche bedeutende Chir urgen gegen sich. So trepanirt L u c as Cha mp i on nièr'e noch mit der Krolle; die Vereinigung zweier Trepanöffnungen bewirkt el mit Hammer und Meissel (s. z. B. Archives méd. Belges, März 1889 und XI. internationaler Congress 1894); ebenso empfiellit Cant, Lancet, 7. März 1891, die Trephine; in England und Amerika sind bei einer grossen Zalıl von Chirurgen - ich nenlle nur Horsley - Kronen mit grosser Oeffnung in Gebraucl. Auch bei uns wird bei intactem Schädeldach der Trepan noch vielfacl benutzt (z. B. Czerny, s. B. v. Beck jun., Beiträge zur Pathol. u. Chir. des Gehirns, S. 101, Tübingen 1894; Kü ster, Freic Vereinigung der Chirurgen Berlins 1888, u. a. m.)

Von Modificationen des Trepans ist der: etwas ältere, voll Stoll (Warschau) empfohlene "Rychanotrepan" (s. Centralbl. für Chir. 1886, No. 28 und 1889, No. 27) zu erwähnen, welcher, ähnliclı dem alten Exfoliativtrepan, die Oeffinung heraushobelt, dabei abel. die Knochensplitter in sich aufnimmt; ferner der von Tauber in Centralbl. f. Chir. 1892, No. 20 empfohlene Torne-Trefin: dieser sägt kegelförmige Stïcke aus, welche nachher gut aufliegen, nicht in die Lücke hineinsinkell. Neue Instrumente (Trepan) für die temporäre Resection empfiehlt $\mathrm{Zuccal} \cdot \mathrm{r}^{\circ}$ Gazz. di Torino, 1890. Zur' Erweiterung von 'Trepanlïcken wird am häufigsten der Meissel benutzt; v. Bardeleben hat zu diesem Zweck an der einen Ecke des Meissels eine kurze stumpfe Sonde anbringen lassen, welche beim Meisseln zwischen Knochen und Dura liegt und diese schützt. Mit der Luer'schen Hohlmeisselzange, nit dem Pince-Trepan Faraboeuf's, dem Craniotom Poirier's und Delvoie's (l. c. S. 71), mit dem Meisselmesser und der gedeckten Säge Thomalla's (Centralbl. f. Chir. 1892, No. 41), dem "Winkelmeissel" von Lalle, einer Modification des Hohlmeissels (London 1893), der Kreissäge von Salzer (Wien. klin. Wochenschr. 1889, No. 49 und Knox, Glasgow med. Journ., April 1893) und einer Modification desselben, der Trepansäge von Ärendt (XX. Chirurgencongress 1893) können entweder bestehende Oeffnungen erweitert oder, z. B. für die temporäre Resection, das abzuhebende Knochenstück schneller und sicherer "umschnitten" werden, als es mit Hammer und Meissel alleill möglich ist. Freilich lat der letztere die grössere Einfachheit für sich, und mit der Gefahr des "Verhämmerns" scheint es nacl den Erfahrungen voll Wagner (Königshütte), Czerny, Lauen stein, denen sich wohl die Mehrzahl der Cliirurgen anschliesst, nicht so schlimm zu sein. - Allgemein üblich, wenn auch nicht immer besonders erwähnt, ist es wohl, mit den Meissel schräg zu arbeiten, so dass, wie bei dem Torne-Trefin Tauber's, die Ränder nachher aufeinander liegen. In der v. Bardeleben'schen Klinik ist es immer so gemacht, ebenso operirte Bellda (siehe oben) auf diese Weise; ganz besonder's empfohlen wird das Verfahren von Metzler v. Andelberg (Wien. klin. Wochenschr. 1890, No. 42) und von C. Beck (Centralbl. f. Chir. 1894, No. 44). - Eine feine Säge benutzte fül die temporäre Resection auch Toison (Revue de chir. 1891, No. 5 und Journ. méd. de Lille 1890, No. 45). Ein etwas complicirtes Instrunent zur diagnostischen Anbohrung des Schädeldaches empfiehlt Bradford in Boston (s. Ref. im Centralbl. f. Chir. 1894, No. 52)

Ueber die Methoden der Trepanation ergiebt sich inanches schon aus der vorstehenden Aufzählung neuer Instrumente. Wir köınen die Methoden eintheilen in solche, bei denen ein Stück aus dem Schädeldache entfernt wird - das wäre die alte gewölnnliche Trepanation und ihre moderne Verwandte, Craniectonie, und in solche, bei denen eine "Klappe" aus Weichtlleilen und Knochell gebildet und durch Umlegen derselbell nach aussen die 
Schädelhöhle nur für die Zeit der Operation geöffnet wird - das ist die osteoplastische temporäre Resection. Bei dieser entsteht kein Defect; bei jener kann der entstandene Defect auf sehr verschiedene Weise verschlossen werden (Cranioplastik); durch Reimplantation der heraustrepanirten Scheibe, durch "Pflastern" der Dura mit den Stückchen desselben oder fremden Knochens, durch Lappenverschiebung (Müller, König), durch Einpflanzen anderer Substanzen, z. B. von Celluloidplatten. Beabsichtigt man eine dauernde Druckentlastung, dann wird man auf den Verschluss der Knochenlücke lieber verzichten, so z. B. bei der Craniectomie wegen Mikrocephalie, auch zuweilen bei Trepanationen wegen Jackson'scher Epilepsie. Wie Lucas Championnière mittheilte, hat Verchère in einem derartigen Falle ohne Reimplantation einen sehr günstigen Erfolg erzielt (Bullet. de la Soc. de chir. XVII). Natürlich wird man auch bei Hirnabscessen nicht an den sofortigen Verschluss der Trepanlücke denken.

Eine Lücke in den Schädelknochen kann bei unversehrter Dura, besonders bei jungen Personen, auch ohne Osteoplastik, im Laufe der Zeit einen knöchernen Verschluss bekommen, wie in neuerer Zeit u. a. ein von Carl Beck (Chicago), in seiner interessanten Arbeit: „Cranioplastic operations" mitgetheilter Fall beweist (The Journal of the Amer. med. Assoc., 15. December 1894 ; vergleiche auch Konrad, Würzburg, 1889: Ueber Regeneration der Knochendefecte des Schädels nach Trepanation). Dass man aber oft dahin gestrebt hat, diese Lücken möglichst bald durch einen festen Verschluss zu sichern, wird durch manche früheren Versuche (s. v. Bergmann's Kopfverletzungen, S. 557) und altere Andentungen bewiesen. - Trepanlöcher, viereckige und runde, sind übrigens in zahlreichen Gräbern aus der Steinzeit gefunden. ${ }^{1}$ ) In neuerer Zeit ist die Wiedereinpflanzung sehr häufig ausgeführt in Thierversuchen wurden die feineren Vorgänge bei der oft erreichten glatten Einheilung untersucht, von einzelnen wurden Modificationen der einfachen Einpflanzung empfohlen. So machte Clark (Lancet, 6. Februar 1886, Trephining in epilepsy) die $3 / 4$ Zol dicke Scheibe dünner, ehe er sie reponirte, und bohrte daneben zur Drainage ein kleines Loch für einige Catgutfüden. Cunes (Rif. med. 27. Janluar 1891) machte die untere Fläche möglichst glatt, um Verwachsungen mit der Dura zu vermeiden; Walker (Med. Rep. 1890, No. 9) ist gegen alle Manipulationen der Art, weil er danach erst recht die Bildung von Osteophyten befürchtet. Interessant sind in dieser Beziehung die Versuche von Sacch (Rif. med., 4. August 1893), nach welchen keine Verwachsung mit der Dura eintritt, wenn die Scheibe umgekehrt, das Periost nach innen eingepflanzt wird. Ausgedehnte Thierversuche stammen von Guérin (Bull. de l'Acad. 1888, No. 44); casuistische Mittheilungen von Pous (Thèse de Lyon 1891), Burrell (Boston med. Journ. 29. März 1888), George St. George (Brit. med. Journ. 24. April 1886, S. 779), Hoegh (Med. News 1890, No. 8), Sawtelle (s. Centralbl. f. Chir. 1893, No. 24), Olmstedt (Ann. of surg. Nov. 1892, das "Pflastern" der Dura mit zahlreichen Stückchen der zerkleinerten Scheibe), Gerstein (Chirurgencongress 1889, Einheilen eines ganz gelösten Stückchens). Aehnlich verfuhr Körte in einem Fall von offenem Schädelbruch (Berl.klin. Wochenschr. 1893, No. 31) und Jack son (Boston med. Journ. 23. März 1893). Ueber die von Kümmell und später von Senn empfohlene Einpflanzung von decalcinirten Knochen haben Darkschewitsch und Weidenh a mmer (Ref. Centralbl. f. Chir. 1892, No. 41), Versuche angestellt. - Zahlreich sind die Mittheilungen über Heteroplastik, speciell über die Einpflanzung fremden, unorganischen "todten" Materials: A. Fraenkel berichtete über gelungene Thierversuche mit Celluloid (Wien. klin. Wochenschr. 1890, No. 25 und in Bruns Beitr., Festschrift für Billroth). Hinterstoisse1 (ebenda, 1891, No. 16 und 1894, No. 4), v. Eiselsberg (Internat. klin. Rundschau 1891, No. 14 und XX. Chirurgencongress), v. Frey (Wien. klin. Wochenschr. 1894, No. 3) und Fill enbaum beobachteten das Einheilen von Celluloidplatten in Schädeldefecten. Winiwarter (s. Delvoie, l. c. S. 314) musste in einem seiner Fälle die Celluloidplatte wegen Eiterung wieder entfernen: auch bei Berger (Bull. de la Soc. de chir. XVIII) misslang diese Heteroplastik; es trat eine Ansammlung von stinkendem serösem Eiter unter der Scheibe ein. (Vergleiche auch Mosse, Sem. méd. 1893,

1) Munro, Prähistorische Trepanationen. s. Centralbl. f. Chir. 1894 No. 27. - Horne, Trepanation sonst und jetzt. London 1894. - Mc S e e primitive trephining (Inkaschädel). Hopkins Hosp. Bull. 1894. - Horsley Brit. med. Journ. 1889 I, S. 456, demonstrirte Schädel aus der Steinzeit mit Trepanationsöffinungen uber der motorischen Region. - Cartailhac, Crânes trépanés à l'age de la pierre, Annales méd. Jan. 1888; Trépanation préhistorique. Hospital Bullet. de l'Acad. de Clermont, Sept. 1890 - Auch Broca hat uber interessante derartige Befunde berichtet; vergl. P. Delvoie, La trépanation cranienne, Brüssel, 1893, wo noch die Arbeiten von Prumières, de Baye, L. Championnière, Albert, Rose, Seydel, Albu citirt sind.
S. 250 und Le Dentu, ebenda S. 51.) - Auch die Versuche mit anderem Material, mit Gips, Guttapercha, Lack, haben, wie wir aus der schon citirten Arbeit von C. Beck in Chicago sehen, wenigstens bei Thierversuchen schon zu recht bemerkenswerthen Resultaten geführt. Wir wollen hierbei auf die interessante Notiz hinweisen, welche sich bei H. Dumas (Thèse de Paris 1889) findet, dass bei den Bewohnern der Südseeinseln die Trepanationsöffnungen durch dünne und glatte in die Lücken hineingepasste Scheiben von der Cocosnuss verschlossen werden.

Von Arbeiten, welche die verschiedenen Methoden der Cranioplastik besprechen, nennen wir: Moiss on, Des differentes méthodes d'obliteration des pertes de substance du crâne (viel Litteratur!). Thèse de Paris 1891. - Chipault, L'ostéoplastie cranienne. Gaz. des hốp. 1893, No. 83. Codivilla, Ueber die Mittel, Schädeldefecte zu schliessen. Arch. di ortoped. 1892 (ist für die temporäre Resection, also eigentlich für Verhitung des Defectes). - Brentano, Ueber traumatische Schädeldefecte und ihre Deckung. Deutsche med. Wochenschr. 1894, No. 17. - Sacchi, Beiträge zur Schädelplastik. XI. intern. Congress und Rif. med. 1894, 7. Juni.

Wo es sich nicht um die Herbeiführung einer dauernden Druckentlastung oder einer längeren Drainirung handelt, da ist auch keine eigentliche Craniectomie, folglich auch keine spätere Osteoplastik nöthig; in diesen Fällen wird immer mehr die sofortige Autoplastik, die temporäre Resection, ausgeführt. Die dafür von Wagner (Königshütte) gegebenen Vorschriften (s. Centralblatt für Chirurgie 1889, No. 47 und 1891, No. 2) gelten der Hauptsache nach noch heute; die Zahl der nach dieser Methode ausgeführten temporären osteoplastischen Schädelresectionen ist trotz der wenigen Jahre seit der ersten Empfehlung eine ganz beträchtliche. Von den Modificationen beziehen sich einige auf das Fortlassen der kurzen Schenkel an der Basis des Lappens, andere machen diesen viereckig und benutzen besondere Instrumente zur Bildung des Knochenlappens (To is on, Zuccaro, Lane, Krouchkoll, Paris 1893, Tauber, C. Beck u. s. w.; s. o.). Zwei von den neuen Vorschlägen für die temporäre Resection scheinen uns besonders wichtig und interessant; einer von Müller (Centralblatt für Chirurgie 1890, No. 4) welcher nicht die ganze Dicke, sondern nur die äusserste Schicht des Schädelknochens mit in den Lappen nimmt und die übrigen Schichten herausmeisselt; der andere von Scafi, Florenz 1893, auch empfohlen von Tassi (Ref. Centralblatt für Chirurgie 1893, No. 31), welcher an der Briicke des später zu bildenden Lappens in der ersten Sitzung Haut und Knochen lineär durchtrennt, die Hautwunde vernäht und nach einer Reihe von Tagen, wenn die Weichtheilwunde verbeilt ist, die übrige Operation vornimmt und nun nicht mehr nöthig hat, die Brticke durchzubrechen. Müller war zu seiner, allerdings nicht leicht auszuführenden Modification durch die König'sche Methode der Rhinoplastik gekommen: König selbst hat nun in ganz ähnlicher Weise für manche Fälle von Schädeldefecten die Deckung durch einen, der Nachbarschaft entnommenen, Haut, Periost und obere Knochenschicht enthaltenden, gestielten Lappen vorgeschlagen und ausgeführt. Der neue, bis in die Diploë reichende Defect wird, so weit es geht, durch die abgelöste Bedeckung des alten, ebenfalls in Form eines gestielten Lappens, oder durch Transplantationen bedeckt. (Centralblatt für Chirurgie 1890 , No. 27.) Zahlreiche günstige Erfahrungen beweisen die Leistungsfähigkeit dieser Art Cranioplastik: Ri e gner, Deutsche medicinische Wochenschrift 1893 , No. 28 (1 Fall); Schoenborn, XX. Chirurgencongress (1 Fall) Tietze-Mikulicz, XXI. Chirurgencongress (2 Fälle); Kehr. Centralblatt für Chirurgie 1893, No. 48 (1 Fall); Borelius, ebenda No. 10 (2 Fälle); Slajmer, Wiener klinische Wochenschrift 1893, S. 213 (1 Fall)

Ungefähr gleichzeitig mit diesen osteoplastischen Operationen, welche an Thieren von J. Wolff schon beinahe 20 Jahre vorher erprobt, aber nicht weiter verfolgt waren (s. Centralblatt für Chirurgie 1890 , No. 1), trat Lannelongue mit dem Vorschlage auf die Mikrocephalie und ihre Folgezustände operativ anzugreifen (Union méd. Juli 1890, Académie des Sciences méd. Juni 1890 VII. französischer Chirurgencongress 1891). - Zwei Meinungen sind es, welche einander hier gegenüberstehen: nach der einen (Bourneville, Ballet, Régis, Bouchereau, Rouby, Gaz. des hôp. 1892, No. 152 und Progr. méd. 24. Juni 1893; Benedikt, Wiener medicinische Presse, 10. Juli 1892, Wal de yer u. a.) ist die mangelhafte Entwickelung des Gehirns das Primäre und die Mikrocephalie ist nur eine Folge davon - dann würde eine Operation wenig $Z$ weck haben. Nach der anderen Meinung wird die Entwickelung des Gehirns durch eine frühzeitige Verknöcherung und Wachsthumshemmung des Schädels aufgehalten - dann würde in der Craniectomie (oder Craniotomie) das einzige Mittel zur Besserung liegen. Beide Fälle sind denkbar; die Schwierigkeit liegt nur darin, sie zu unterscheiden, und diese Schwierigkeit wird dadurch nicht geringer, dass man von einer angeborenen und einer erworbenen Mikrocephalie spricht. Es wird gar nicht so selten vorkommen, dass man nicht entscheiden kann, welche Form man 
vor sich hat; dann ist man auf Grund der zahlreichen, bisher veröffentlichten Erfolge voll berechtigt, die Operation vorzunehmen. Sie ist allerdings gefährlicher als Schädeloperationen aus anderen Ursachen; oft wurde Shok während des Meisselns, zuweilen der Tod kurz nach der Operation beobachtet. Dafïr sind aber auch eine ganze Reihe von Besserungen dieses sonst ganz hoffnungslosen Zustandes durch den Eingriff verzeichnet. Wenn Akermann (Ueber die operative Behandlung der Mikrocephalie, Volkmann's Sammlung klinischer Vorträge, Neue Folge, No. 90) 13 Todesfälle bei 75 , nach v. Bergmann's Anmerkung auf S. 547 bei ungefähr 70 Operirten, zusammenstellt, so ist dabei zu berücksichtigen, dass diejenigen Fälle selten sind, in denen gar kein Erfolg erzielt wurde ${ }^{1}$ ). - Die operative Technik bei diesem Eingriff ist sehr verschieden gewesen; Lan nel ongue selbst hat seine lineäre Craniektomie später durch lappenförmige Schnitte er'setzt, um grössere Stücke des Schädeldaches mobil zu machen; Mac K lin tock (Centralblatt für Chirurgie 1892, No. 12) legte in jedem Seitenwandbein eine Trepanöffnung an und schnitt von hier ans subcutan nach vorn und nach hinten durch den Knochen hindurch; Postempsky umschnitt ein Viereck, liess aber in den Weichtheilen an den vier Ecken eine Brücke stehen, während der Knochen vollständig durchschnitten wurde; Bauer (Ref. Centralblatt für Chirurgie 1890, No. 44) legte an jedem Seitenwandbein zwei Trepanoffnungen an und durchsägte die Brïcke zwischen ihnen; J aboulay (Arch. prov. de chir. II) meisselte zwei parallele Rinnen und durchtrennte subcutan die Knochenbrïcke an beiden Enden der Schnitte (Trépanation bilinéaire); Gersuny löst das ganze knöcherne Schädeldach $a b$ in der Art, wie es bei Sectionen iiblich ist (Wiener medicinische Presse 1893, No. 3). Hier, wo es sich darum handelt, die gesammte Entwickelung des Gehirns zu erleichtern, wird man natürlich alles vermeiden, was einen frühzeitigen Verschluss der angelegten Spalten begünstigen könnte; man wird also das Periost, soweit es den Spalt überbriicken würde, mit fortnehmen. Die Dura wird, wenn sie gesund ist, nicht berührt; die Oeffnung im Knochen wo möglich so gemacht, dass sie der Hautwunde nicht ganz entspricht (Tillmanns u. a.).

Wenn wir schon bei der chirurgischen Behandlung der Idiotie nur selten auf klare Anzeigen hin operiren konnten, so ist dies noch seltener der Fall bei Psychosen und bei der allgemeinen Paralyse. Ist der Zusammenhang einer Geisteskrankheit mit einer bestimmten Kopfverletzung nachgewiesen und deuten Veränderungen am Schädel oder bestimmte Heerdsymptome auf eine Stelle, welche ähnlich wie bei der traumatischen Epilepsie als Ausgangspunkt betrachtet werden kann, dann ist der Eingriff wohl als berechtigt anzusehen und hat auch mehrfach schon bedeutende Besserung herbeigeführt. So in dem von Stetter operirten Falle (Centralblatt für Chirurgie 1892, No. 20). Flührer beobachtete Heilung einer Geisteskrankheit nach Trepanation und Entleerung einer Cyste unterbalb einer Narbe an der Stirn (Schussverletzung drei Jahre vorher, s. Lancet 1. Mai 1886). In dem von Keen (Amer. Journ. of med. Sciences, September 1891) berichteten Falle war der Erfolg nur vorübergehend. In dem bekannten Falle, über welchen Durante auf dem Congress in Washington (Lancet, 1 . October 1887, S. 654) berichtete, handelte es sich um eine früher lebenslustige Person, welche während der Entwickelung eines Tumors

1) Zu A kermann's Litteraturangaben sind noch beizufügen: Revel, Beitrag zur Hirnchirurgie. Trepanation und Craniektomie bei Mikrocephalie, Hydrocephalie und Epilepsie. Thèse de Montpellier 1892. - Tillmanns, Ueber Craniektomie bei Mikrocephalie. 23. Congress der Deutschen Gesellschaft für Chirurgie 1894 (2 Fälle ohne wesentliche Besserung). - P hi lip p on, Ueber Craniektomie bei Mikrocephalie. Lyon 1893. - P os te m psky, Neues Verfahren bei Osteotomie wegen Mikrocephalie. Rif. med. 4. März 1893. - Ceresole, Craniektomie bei Mikrocephalie. Thèse de Lausanne 1894. - Akermann (s. o.), Ein Fall mit deutlicher Besserung nach der Operation. Congr. d. Chir. des Nordens, Sem. méd. 1893, S. 359. Nuijens, Eine Craniectomie bei Mikrocephalie. Ann. d'Anvers Juni 1893 (Kein Erfolg). - Rie, Eine Craniektomie bei Mikrocephalie nach Gersuny. K. K. Gesellsch. d. Aerzte, 13. Jan. 1893. - Reboul, Craniektomie bei Mikrocephalie; Cyste in der vorderen Schädelgrube; Besserung der cerebralen Functionen nach der Operation. Arch. prov. de chir. II. - Dumont, Die circuläre Craniektomie. Correspondenzblatt f. Schweiz. Aerzte. 1. Dec. 1893. - Barlow, Ein Fall von Besserung. Glasgow med. Journ., Juni 1893. Angell, Bei einem Kinde mit Hemiplegie, Imbecillität und Epilepsie Besserung durch die Craniektomie. Journ. of mental dis., Oct. 1894. Willard und Lloyd, Trepanation bei Porencephalie, Besserung. Amer. Journ. of med. Sc., April 1892. -- Eng e I, Med. News 23. April, Med. Rec. 4. Juni 1892, berichtet über einen Fall von Besserung bei Dementia epileptica nach linearer Craniektomie. - Winiwarter (s. Delvoie, l. c., S. 310) verftigt über 7 Fälle; einmal Tod am Tage der Operation an intracranieller Blutung; zweimal kein, zweimal geringer und einmal sehr guter Erfolg; allerdings erst kurze Zeit beobachtet. Winiwarter implantirte nach der Craniektomie mehrere male Celluloidplatten in dio Lücke. Masson, Beitrag zum Studium des Verfahrens bei der Craniektomie. Lyon 1894. im Stirnhirn mclancholisch, reizbar und gedächtnissschwach geworden war und nach Entfernung des Tumors ihre frühere Sicherheit und gute Stimmung wiederbekam. Wir können uns auf die kurze Hindeutung beschränken, da bei diesen opcrativen Eingriffen eine besondere Technik nicht erforderlich ist. Beschaffenlıeit, Stelle und Ausdehnung einer Narbe, einer Depression, Sitz und Grösse einer angenommenen intracraniellen Läsion sind für das operative Vorgehen maassgebend. (Vergl. Burckardt, Zeitschrift für Psychologie Bd. 47.)

Ander's steht es mit der letzten der uns hier interessirenden Operationen, mit der Entleerung der Hirnventrikel bei zu starker Ansarnmlung oder Spannung des Liquor cerebrospinalis. Hier handelt es sich um ganz bestimmte Stellen, an denen der Eingriff beginnt; ähnlich wie bei der Aufsuchung der zwei Hauptäste der Arteria meningea media nach $\mathrm{Kr}$ önlein (Deutsche Zeitschrift für Chirurgie Bd. 23). Die Vorschriften des letzteren sind neuerdings von Steiner (Langenbeck's Archiv Bd. 48) etwas modificirt; dieser legt die vordere Oeffuung etwas höher an, um diejenige Stelle zu vermeiden, an welcher die Arterie oft durch einen knöchernen Canal geht. Dass diese Stelle bei Krönlein's (auch Voigt's) Methode oft getroffen wird, habe ich bei Operationscursen in einer grossen Zahl von Fällen demonstriren können. $O b$ das einen grossen Nachtheil, z. B. bei der Entleerung lebensgefährlicher Hämatome bildet, ist eine andere Frage; trotzdem kann man nach Steiner's Vorschlag diese Oeffnung etwas höher anlegen - sofern dadurch nicht die Entleerung des Hämatoms erschwert wird. Uebrigens habe ich den mittleren, auch recht stattlichen Ast bei Operationscursen oft freilegen lassen; die Oeffnung liegt in derselben Höhe wie die für den vorderen und hinteron Ast, vertical über dem äusseren Gehörgang. - Wenn man bei diesen Operationen die temporäre Resection anwendet (vielleicht mit secundärem Verschluss der Oeffnung), dann ist eine genaue Localisation auch hier nicht nöthig.

Für die Punction der Ventrikel, für welche wir in v. Berg mann's chirurgischer Behandlung der Hirnkrankheiten einen interessanten Belag und eingehende Betrachtungen finden, geben Kocher, Delvoic (nach Winiwarter), Poirier, Keen, Le Fort u. a. specielle Vorschriften. Diejenige Stelle, an welcher die Dicke der bedeckenden Hirnschicht am geringsten ist, befindet sich in gleicher Höhe mit den Stellen für die Arteria meningea media, ungefähr in der oben genannten, für den mittleren Ast maassgebenden Partie (ganz wenig nach hinten davon). Kocher hat diese Stelle, weil ihm hier einmal die Drainage versagte, nicht wieder gewählt und den Ventrikel lieber von oben und vorn her, in der Furche zwischen erster und zweiter Stirnwindung, ca. $3 \mathrm{~cm}$ lateral von der Mittellinie geöffnet, obgleich er hier durch eine 4-5 cml dicke Schicht hindurch punctiren musste; dieselbe Stelle hatte v. Bergmann gewählt. Keen, Broca, Poirier n. a. empfehlen die laterale Punction. Dem oben erwähnten Vorschlage von M. Schmidt für die diagnostische Hirnpunction entspricht Sahli's Trepanopunction zur Entleerung der Ventrikel; er macht die Oeffnung im Schädel nur so gross, dass nachher die Canüle darin Platz hat, welche zur weiteren Entleerung liegen bleibt. (Klinische Vorträge 1891, No. 28). Kocher (Chirurgische Beiträge II, s. o.) äussert sich günstig ïber die Aussichten der Ventrikelpunction bei allgemeiner Epilepsie und Porencephalie; ebenso Wy s s (Correspondenzblatt für Schweizer Aerzte, 15. April 1893). Beide empfehlen langdauernde Drainage (vergl. auch die Operationslehre von Kocher, S. 38), wïhrend Diller (Amer. Journ. Nov. 1892) sich gegen die Drainage ausspricht. Baskelt (Brit. med. Journ. 13. Jan. 1894) beobachtete nach der Punction und Drainage bei Hydrocephalia chronica eine Besserung, welche drei Monate anhielt, dann erfolgte der Tod durch Meningitis. Keen (Med. News, 1. December 1888) giebt genaue Vorschriften für die Operation. Weitere Arbeiten über dieses Thema lieferten: Boltze (Halle 1893), Ein Beitrag zur operativen Behandlung des Hydrocephalus congenitus; Vautrin (Sem. méd. 1893 S. 377) Ueber die Behandlung des Hydrocephalus congenitus; Manny (ebenda S. 170), Trepanation in einem Falle von Hydrocephalus chronicus (Tod nach fünf Tagen); Broca (Rev. de chir. 1891, No. 1): Die Drainage der Hirnventrikel bei Hydrocephalus (vergl. Gaz. hbd. 1891, No. 15, Zwei Fälle von Trepanation bei Hydrocephalus internus, 1 ); Phocas (Rev. des mal. de l'enfance, Februar 1892), Chirurgische Behandlung des Hydrocephalus; Parkin (Lancet, 1. Juli 1893), Ueber das Ablassen von Cerebrospinalflüssigkeit aus dem subarachnoidalen Raum, um den intracraniellen Druck herabzusetzen. Hier ist auch die von Quincke (Berliner klinische Wochenschrift 1891, No. 38 und 39) empfohlene Lumbalpunction bei Hydrocephalus zu nennen.

Wie aus unserer Darstellung hervorgeht, bilden die zuletzt besprochenen Hirnkrankheiten ein noch nicht ganz gesichertes Gebiet für unsere operativen Eingriffe; ebenso verhält es sich mit 
der allgemeinen Epilepsie ${ }^{1}$ ); sie ist der Trepanation zugänglich, wenn sie sich in ihren Symptomen der Jackson'schen Form nähert, wenn sie auf bestimmte erreichbare Centren zu beziehen ist. In derselben Weise sind die heftigen, jeder Behandlung trotzenden und mit Wahrscheinlichkeit auf erhöhten intracraniellen Druck zu beziehenden Kopfschmerzen zu beurtheilen. Bei der operativen Behandlung dieser Zustände, zu denen wir noch die Apoplexieen rechnen können (s. Braddo n, Lancet 1887, 15. Oct.), muss, wie v. Bergmann am Schluss seines öfter eitirten Werkes sagt, mit viel Kritik und viel Vorsicht gearbeitet werden, wenn sie einen wirklichen und bleibenden Fortschritt bedeuten soll.

(Fortsetzung folgt.) 